\title{
Risk factors for adjacent segment degeneration after posterior lumbar fusion surgery in treatment for degenerative lumbar disorders: a meta-analysis
}

Tao Wang ${ }^{1}$ and Wenyuan Ding ${ }^{2^{*}}$

\begin{abstract}
Study design: A meta-analysis.

Objective: We performed a meta-analysis to explore the incidence and risk factors of adjacent segment degeneration (ASD) after posterior lumbar fusion surgery.

Methods: An extensive search of the literature was performed in English database of PubMed, Embase, and Cochrane Library, and Chinese database of CNKI and WANFANG (up to May 2020). We collected factors including demographic data, surgical factor, and sagittal parameters. Data analysis was conducted with RevMan 5.3 and STATA 12.0.

Results: Finally, 19 studies were included in the final analysis. In our study, the rate of ASD after posterior lumbar fusion surgery was 18.6\% (540 of 2896). Our data also showed that mean age, body mass index (BMI), the history of smoking and hypertension, preoperative adjacent disc degeneration, long-segment fusion, preoperative superior facet violation, high lumbosacral joint angle, pre- and post-operative L1-S1 sagittal vertical axis (SVA), post-operative lumbar lordosis $(\mathrm{LL})$, and preoperative pelvic incidence (PI) were associated with the development of ASD. However, gender, history of diabetes, bone mineral density (BMD), preoperative Oswestry Disability Index (ODI) and Japanese Orthopedic Association (JOA), the type of fusion (PLIF vs TLIF), type of bone graft (auto- vs allograft), fusion to S1 (vs non-fusion to S1), diagnose (lumbar disc herniation, lumbar spinal stenosis, lumbar spondylolisthesis), preoperative pelvic tilt (PT), LL and sacral slope (SS), post-operative SS, PT and PI were not associated with the development of ASD.
\end{abstract}

Conclusions: In our study, many factors were correlated with the risk of ASD after posterior lumbar fusion surgery. We hope this article can provide a reference for spinal surgeons in treatment for lumbar degenerative diseases.

Keywords: Incidence, Risk factors, Adjacent segment degeneration, Lumbar, Fusion surgery, Posterior, Meta-analysis

\section{Introduction}

Due to good clinical results, posterior lumbar fusion surgeries have been widely used in treatment for various lumbar degenerative diseases. Though the initially good clinical results after fusion, biomechanical change of the

\footnotetext{
* Correspondence: docwangspine@163.com

${ }^{2}$ Department of Spinal Surgery, The Third Hospital of Hebei Medical University, No. 139 Ziqiang Road, Shijiazhuang 050051, China

Full list of author information is available at the end of the article
}

spine caused by fusion may accelerate the degeneration of the adjacent segment [1]. So, adjacent segment disease or adjacent segment degeneration (ASD) is considered to be a potential long-term complication of spinal fusion. The rate of ASD, considered radiographic changes without symptom, ranges from 5.2 to $49 \%$ in various studies after posterior lumbar fusion surgery [2].

Some researchers found that ASD may be caused by lumbar fusion, which can induce abnormal intradiscal 
pressure and too much movement at the adjacent spinal levels, resulting in abnormal discal stress distribution [3, 4]. However, Battie et al. [5] found that ASD after fusion was a natural process that was not related to fusion surgery. Recent articles have reported the risk factors for ASD including older age, female, expression of the estrogen receptor, the number of instrumented level, preexisting degenerative condition at an adjacent motion segment, sagittal alignment change [6-10]. As far as we know, the risk factors for ASD remain controversial. Therefore, this study aims to explore the incidence and risk factors of ASD following posterior lumbar fusion surgery for degenerative lumbar disorders.

\section{Methods}

\section{Search strategy}

We searched for the English and Chinese language studies with the keywords: "adjacent segment degeneration" or "ASD", and "lumbar surgery" in English database of PubMed, Embase, and Cochrane Library and Chinese database of CNKI and WANFANG. There was no limitation on the date of publication, which covered all previously published studies up to May 2020.

\section{Eligibility criteria}

Included articles must satisfy: (1) study population must be adult patients; (2) measured outcomes of the incidence and risk factors of ASD; (3) comparison: ASD group and non-ASD group; (4) the study must be meet the definition of ASD (defined as a radiological change in which narrowing of disc height was $\geq 3 \mathrm{~mm}$, the progressive slipping of adjacent segments was $\geq 3 \mathrm{~mm}$ (in comparison with preoperative flexion and extension lateral radiographs), and the posterior opening of adjacent segments was $5^{\circ}$; (5) follow-up of more than 2 years; and (6) patients with lumbar disc herniation, lumbar spinal stenosis, lumbar spondylolisthesis. Studies were excluded if they (1) were abstracts, letters, reviews, or case reports; (2) had repeated data; (3) did not report outcomes of interest; (4) patients treated for lumbar trauma, tumor, infection, inflammation, scoliosis; and (5) patients underwent any other lumbar surgery.

\section{Data extraction and outcome measures}

The data included the general characteristics of each study and the outcomes measured. General characteristics included first author, year of publication, country, the number of ASD patients and total patients, follow-up time, type of article, shown in Table 1. The outcomes include the rate of every risk factors. When the same population was reported in several publications, we retained only the most informative article or complete work to avoid duplication of information.
Data were extracted independently by two authors. Any disagreements concerning paper eligibility were resolved by discussion and consensus. Test for risk of publication bias. We performed a visual inspection of the funnel plot for publication bias. The funnel plot should be asymmetric when there is publication bias and symmetric in the case of no publication bias. We performed Egger and Begg tests to measure the funnel plot asymmetry using a significance level of $p<$ 0.10 . The trim and fill computation was used to estimate the effect of publication bias. Sensitive analysis overall because of the low heterogeneity of every factor, so we do not calculate sensitive analysis.

\section{Statistical analysis}

Only dichotomous outcomes were mentioned in our study, so odd ratios (OR) and 95\% confidence intervals (CI) were calculated for outcomes. A $p$ value $<$ 0.05 was judged as statistically significant. Randomeffects or fixed-effects models were used depending on the heterogeneity of the studies included. Heterogeneity was analyzed with both the chi-squared test $I^{2}$ test, where $p$ value of $<0.10$ for the chi-squared and $I^{2}>50 \%$ implied heterogeneity [11]. All statistical analyses were performed using Review Manager version 5.3 (The Cochrane Collaboration, Oxford, UK) and STATA 12.0 (Stata Corporation, College Station, TX, USA).

\section{Results}

\section{Study identification and selection}

Initially, we collected a total of 488 records by the database search. A total of 201 records were excluded due to repetition and 230 records were removed for review based on the titles and abstracts. The remaining 57 records were retrieved for inclusion criteria and 28 of them were excluded, 10 did not report outcomes of interest. Finally, 19 articles that met our inclusion criteria were included in the present meta-analysis. The selection process included in this meta-analysis is shown in Fig. 1.

\section{Baseline characteristics and quality assessment}

The main characteristics of the 19 articles (from 23 to 630 patients) that were published before May 2020 included in the meta-analysis are presented in Table 1. A total of 540 patients were suffering from ASD after posterior lumbar fusion surgery in a total of 2896 patients. According to the 19 included studies, the rate of ASD was $18.6 \%$ (ranged from 8.5 to $69.4 \%)$.

Because all studies included were retrospective studies, we used the Newcastle Ottawa Quality Assessment Scale (NOQAS) to assess the quality of each study. This scale 
Table 1 Characteristics of included studies

\begin{tabular}{|c|c|c|c|c|c|c|}
\hline \multirow[t]{2}{*}{ First author } & \multirow[t]{2}{*}{ Year } & \multirow[t]{2}{*}{ Country } & \multicolumn{2}{|c|}{ No. of participants } & \multirow{2}{*}{$\begin{array}{l}\text { Follow up } \\
\text { time (years) }\end{array}$} & \multirow[t]{2}{*}{ Study type } \\
\hline & & & ASD & Total & & \\
\hline Guoquan Zheng [12] & 2020 & China & 17 & 200 & 7 & Retrospective \\
\hline HUANG LIN [13] & 2017 & China & 40 & 221 & $2-4$ & Retrospective \\
\hline HUANG MI [14] & 2014 & China & 18 & 109 & 2 & Retrospective \\
\hline Hui Wang [15] & 2017 & China & 15 & 237 & $4-6$ & Retrospective \\
\hline Jinqian Liang [16] & 2014 & China & 28 & 84 & 5 & Retrospective \\
\hline Takahiro Makino [17] & 2018 & Japan & 5 & 41 & 2 & Retrospective \\
\hline Yeon Heo [18] & 2015 & Korea & 33 & 378 & 6 & Retrospective \\
\hline Zhao-Ming Zhong [19] & 2017 & China & 18 & 154 & 5 & Retrospective \\
\hline Zhaoxin Ma [20] & 2019 & China & 22 & 71 & $3-6$ & Retrospective \\
\hline Shuta Ushio [21] & 2019 & Japan & 22 & 50 & $2-9$ & Retrospective \\
\hline Seyed Reza Bagheri [22] & 2019 & Iran & 76 & 630 & 7-11 & Retrospective \\
\hline Jun Seok Bae [23] & 2010 & Korea & 11 & 103 & $6-9$ & Retrospective \\
\hline Kyoung-Suok Cho [24] & 2009 & Korea & 9 & 81 & 8 & Retrospective \\
\hline Jaewan Soh [25] & 2013 & Korea & 21 & 55 & 5 & Retrospective \\
\hline Jigar Anandjiwala [26] & 2011 & Korea & 14 & 68 & 5 & Retrospective \\
\hline Masayuki Miyagi [27] & 2013 & Japan & 14 & 23 & 4 & Retrospective \\
\hline Bai-Ling Chen [28] & 2011 & China & 11 & 49 & $2-4$ & Retrospective \\
\hline LI WEISHI [29] & 2018 & China & 50 & 72 & 6 & Retrospective \\
\hline GUO YANG [30] & 2020 & China & 116 & 270 & $3-5$ & Retrospective \\
\hline
\end{tabular}

for non-randomized case-controlled studies and cohort studies was used to allocate a maximum of nine points for the quality of selection, comparability, exposure, and outcomes for study participants. Of these studies, 13 studies scored eight points and 6 studies scored seven points. Hence, the quality of each study was relatively high (Table 2).

\section{Assessment of risk factors of ASD \\ Age}

Ten studies (1560 of 2896 patients) [12-21] reported the age of patients at an operational time between ASD group and non-ASD group. There was no significance in the test for heterogeneity and the studies had low heterogeneity ( $p$ for heterogeneity $=0.43 ; I^{2}=1 \%$, Fig. 2 ). The meta-analysis showed that age was associated with a significant increase in the incidence of ASD (fixed-effects model; $p=0.02, \mathrm{SMD}=1.66,95 \%$ CI $[0.28,3.04]$, Fig. 2).

\section{Body mass index (BMI)}

Four studies (571 of 2896 patients) [12, 15, 16, 21] reported BMI of patients at an operational time between ASD group and non-ASD group. There was no significance in the test for heterogeneity and the studies had low heterogeneity ( $p$ for heterogeneity $=0.62 ; I^{2}=0 \%$, Fig. 2). The meta-analysis showed that BMI was associated with a significant increase in the incidence of ASD (fixed-effects model; $p<0.0001, \mathrm{SMD}=3.17,95 \% \mathrm{CI}$ [2.48, 3.87], Fig. 2).

\section{History of smoking}

Four studies (1250 of 2896 patients) [13, 16, 19, 22] reported a history of smoking between ASD group and non-ASD group. There was no significance in the test for heterogeneity and the studies had low heterogeneity ( $p$ for heterogeneity $=0.24 ; I^{2}=29 \%$, Fig. 2 ). The metaanalysis showed that the history of smoking was associated with a significant increase in the incidence of ASD (fixed-effects model; $p=0.0002, \mathrm{OR}=1.77,95 \% \mathrm{CI}$ [1.24, 2.52], Fig. 2).

\section{Gender}

Fifteen studies (2592 of 2896 patients) [12, 14-27] reported gender between ASD group and non-ASD group. There was no significance in the test for heterogeneity and the studies had low heterogeneity ( $p$ for heterogeneity $=0.89 ; I^{2}=0 \%$, Fig. 3 ). The meta-analysis showed that gender was not associated with a significant increase 


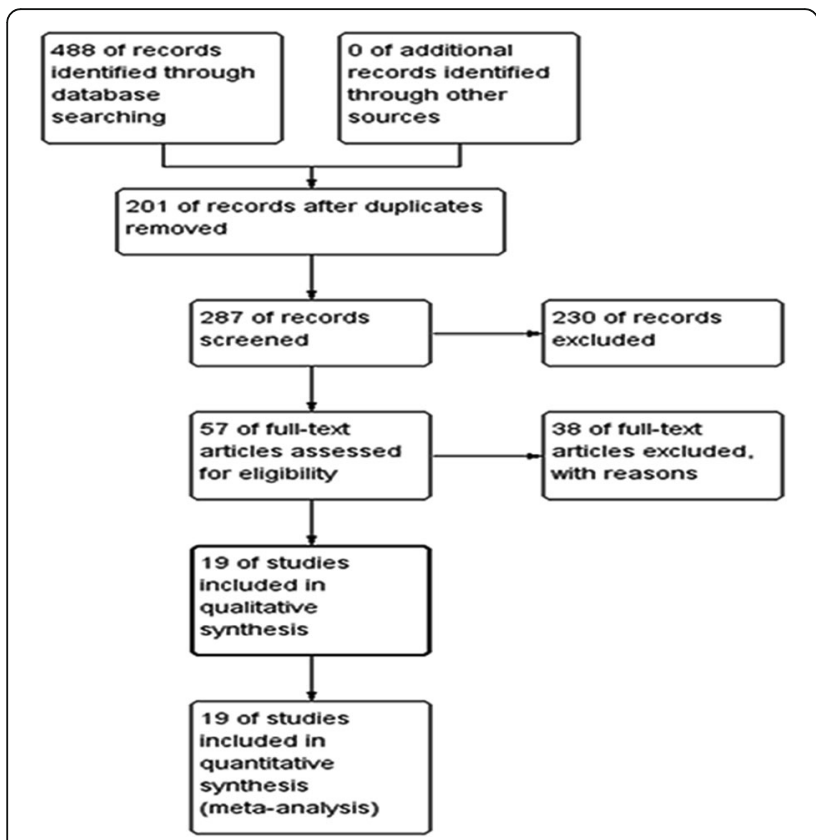

Fig. 1 Flow diagram of the study selection

in the incidence of ASD (fixed-effects model; $p=0.83$, $\mathrm{OR}=0.97,95 \%$ CI $[0.76,1.25]$, Fig. 3$)$.

\section{History of diabetes}

Four studies (1167 of 2896 patients) [12, 19, 21, 22] reported a history of diabetes between ASD group and non-ASD group. There was no significance in the test for heterogeneity and the studies had low heterogeneity ( $p$ for heterogeneity $=0.49 ; I^{2}=0 \%$, Fig. 3 ). The metaanalysis showed that the history of diabetes was not associated with a significant increase in the incidence of ASD (fixed-effects model; $p=0.37, \mathrm{OR}=0.81,95 \% \mathrm{CI}$ [0.51, 1.29], Fig. 3).

\section{Bone mineral density (BMD)}

Two studies (158 of 2896 patients) [14, 28] reported BMD at the operational time between ASD group and non-ASD group. There was not significant in the test for heterogeneity and the studies had low heterogeneity ( $p$ for heterogeneity $=0.31 ; I^{2}=5 \%$, Fig. 4 ). The meta-analysis showed that BMD was not associated with a significant increase in the incidence of ASD (fixed-effects model; $p=0.24$, SMD $=-0.07,95 \% \mathrm{CI}$ $[-0.19,0.05]$, Fig. 4).

\section{Preoperative Oswestry Disability Index (ODI)}

Two studies (120 of 2896 patients ) [20, 28] reported preoperative ODI between ASD group and non-ASD group. There was no significance in the test for heterogeneity and the studies had low heterogeneity ( $p$ for heterogeneity
$=0.41 ; I^{2}=0 \%$, Fig. 4). The meta-analysis showed that preoperative ODI was not associated with a significant increase in the incidence of ASD (fixed-effects model; $p=$ 0.77, SMD $=-0.59,95 \%$ CI [-4.66, 3.47], Fig. 4).

\section{Preoperative Japanese Orthopedic Association (JOA)}

Three studies (161 of 2896 patients) [17, 20, 28] reported preoperative JOA between ASD group and nonASD group. There was no significance in the test for heterogeneity and the studies had low heterogeneity ( $p$ for heterogeneity $=0.42 ; I^{2}=0 \%$, Fig. 4). The metaanalysis showed that preoperative JOA was not associated with a significant increase in the incidence of ASD (fixed-effects model; $p=0.75, \mathrm{SMD}=-0.21,95 \% \mathrm{CI}$ $[-1.54,1.11]$, Fig. 4).

\section{History of hypertension}

Three studies (650 of 2896 patients) [12, 13, 19] reported a history of hypertension between ASD group and non-ASD group. There was no significance in the test for heterogeneity and the studies had low heterogeneity ( $p$ for heterogeneity $=0.31 ; I^{2}=15 \%$, Fig. 5). The meta-analysis showed that the history of hypertension was associated with a significant increase in the incidence of ASD (fixed-effects model; $p=0.001$, OR $=2.29$, 95\% CI [1.37, 3.82], Fig. 5).

\section{Preoperative Pfirrmann's classification at the adjacent segment}

Eight studies (1750 of 2896 patients) [15, 16, 18, 19, 21, 22, 25, 26] reported Pfirrmann's classification between ASD group and non-ASD group. There was no significance in the test for heterogeneity and the studies had low heterogeneity ( $p$ for heterogeneity $=0.49 ; I^{2}=0 \%$, Fig. 5). The meta-analysis showed that preoperative Pfirrmann's classification of more than three levels was associated with a significant increase in the incidence of ASD (fixed-effects model; $p<0.0001, \mathrm{OR}=0.36,95 \% \mathrm{CI}$ $[0.26,0.50]$, Fig. 5).

\section{Short versus long fusion}

Two studies (172 of 2896 patients) [24, 26] reported the length of fusion between ASD group and non-ASD group. There was no significance in the test for heterogeneity and the studies had low heterogeneity ( $p$ for heterogeneity $=0.20 ; I^{2}=14 \%$, Fig. 5). The meta-analysis showed that long fusion $\geq 3$ was associated with a significant increase in the incidence of ASD (fixed-effects model; $p=0.02$, OR $=0.29,95 \%$ CI $[0.10,0.85]$, Fig. 5).

\section{Preoperative superior facet violation}

Two studies (958 of 2896 patients) [15, 22] reported a preoperative superior facet violation between ASD 
Table 2 The quality assessment according to the Newcastle Ottawa Quality Assessment Scale (NOQAS) of each study

\begin{tabular}{lllll}
\hline Study & Selection & Comparability & Exposure & Total score \\
\hline Guoquan Zheng [12] & 3 & 3 & 2 & 8 \\
Huang Lin [13] & 3 & 3 & 2 & 8 \\
Huang Mi [14] & 3 & 2 & 3 & 8 \\
Hui Wang [15] & 2 & 3 & 3 & 8 \\
Jinqian Liang [16] & 2 & 3 & 2 & 8 \\
Takahiro Makino [17] & 3 & 3 & 3 & 7 \\
Yeon Heo [18] & 2 & 2 & 2 & 7 \\
Zhao-Ming Zhong [19] & 3 & 2 & 3 & 8 \\
Zhaoxin Ma [20] & 3 & 2 & 3 & 7 \\
Shuta Ushio [21] & 2 & 2 & 3 & 8 \\
Seyed Reza Bagheri [22] & 3 & 2 & 2 & 7 \\
Jun Seok Bae [23] & 3 & 2 & 3 & 8 \\
Kyoung-Suok Cho [24] & 3 & 2 & 3 & 7 \\
Jaewan Soh [25] & 2 & 2 & 3 & 8 \\
Jigar Anandjiwala [26] & 3 & 2 & 3 & 8 \\
Masayuki Miyagi [27] & 3 & 2 & 3 & 8 \\
Bai-Ling Chen [28] & 3 & 2 & 3 & 7 \\
Li Weishl [29] & 2 & 3 & 3 & 8 \\
Guo Yang [30] & 2 & & & 7 \\
\hline
\end{tabular}

group and non-ASD group. There was no significance in the test for heterogeneity and the studies had low heterogeneity ( $p$ for heterogeneity $=0.53 ; I^{2}=0 \%$, Fig. 6). The meta-analysis showed that preoperative superior facet violation was associated with a significant increase in the incidence of ASD (fixed-effects model; $p<0.00001, \mathrm{OR}=29.74,95 \%$ CI [17.20, 51.43], Fig. 6).

\section{Preoperative lumbosacral joint angle}

Two studies (99 of 2896 patients) [21, 28] reported a preoperative lumbosacral joint angle between ASD group and non-ASD group. There was no significance in the test for heterogeneity and the studies had low heterogeneity ( $p$ for heterogeneity $=0.22 ; I^{2}$ $=34 \%$, Fig. 6). The meta-analysis showed that the preoperative lumbosacral joint angle was associated with a significant increase in the incidence of ASD (fixed-effects model; $p<0.00001, \mathrm{SMD}=-1.87,95 \%$ CI $[-2.50,-1.25]$, Fig. 6).

\section{Type of fusion (PLIF versus TLIF)}

Six studies (650 of 2896 patients) [15, 16, 21, 24-26] reported the type of fusion (PLIF versus TLIF) between ASD group and non-ASD group. There was no significance in the test for heterogeneity and the studies had low heterogeneity ( $p$ for heterogeneity $=0.80$; $I^{2}=0 \%$, Fig. 7). The meta-analysis showed that type of fusion (PLIF versus TLIF) was not associated with a significant increase in the incidence of ASD (fixedeffects model; $p=0.93, \mathrm{OR}=1.03,95 \% \mathrm{CI}[0.61$, 1.73], Fig. 7).

\section{Type of graft (Auto- versus allograft)}

Three studies (232 of 2896 patients) [16, 21, 26] reported the type of graft (auto- versus allograft) between ASD group and non-ASD group. There was no significance in the test for heterogeneity and the studies had low heterogeneity ( $p$ for heterogeneity $=0.34$; $I^{2}=4 \%$, Fig. 7). The meta-analysis showed that type of graft (auto- versus allograft) was not associated with a significant increase in the incidence of ASD (fixed-effects model; $p=0.73, \mathrm{OR}=0.89,95 \% \mathrm{CI}$ $[0.45,1.75]$, Fig. 7).

\section{Fusion to S1 (versus non-fusion to S1)}

Six studies (1071 of 2896 patients) [15, 18, 23, 24, 26, 29] reported fusion to $S 1$ between ASD group and non-ASD group. There was no significance in the test for heterogeneity and the studies had low heterogeneity ( $p$ for heterogeneity $=0.49 ; I^{2}=0 \%$, Fig. 7). The meta-analysis showed that fusion to S1 was not associated with a significant increase in the incidence of ASD (fixed-effects model; $p=0.89$, OR $=0.97,95 \%$ CI [0.60, 1.55], Fig. 7). 


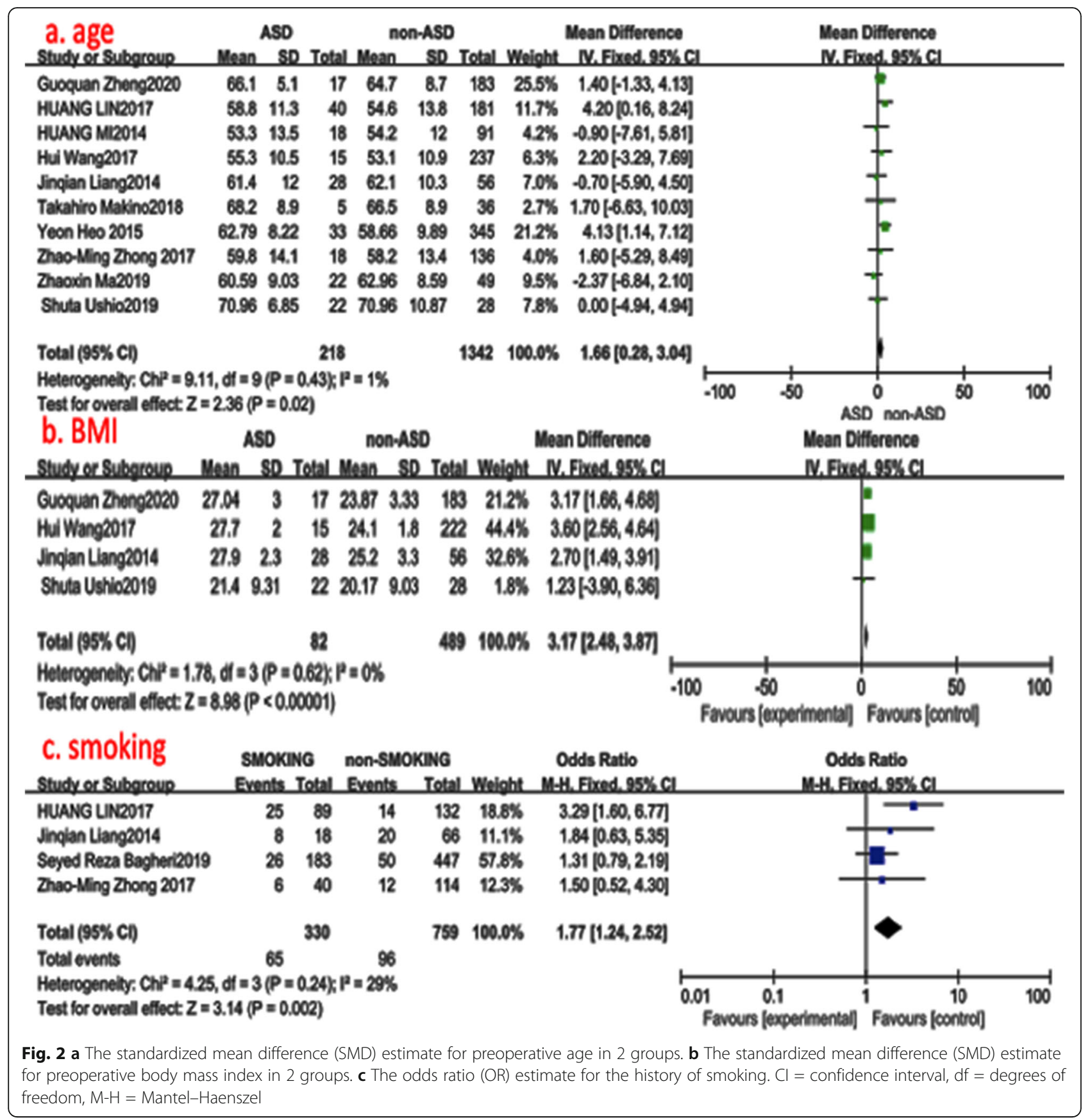

Diagnose (lumbar disc herniation, lumbar spinal stenosis, lumbar spondylolisthesis)

Four studies (392 of 2896 patients) [15, 16, 24, 26] reported diagnose (lumbar disc herniation, lumbar spinal stenosis, lumbar spondylolisthesis) between ASD group and non-ASD group. There was no significance in the test for heterogeneity and the studies had low heterogeneity (three $p$ for heterogeneity $=0.41,0.42,0.24 ; I^{2}=0$, 0, 29\%, Fig. 8). The meta-analysis showed that diagnose (lumbar disc herniation, lumbar spinal stenosis, lumbar spondylolisthesis) was not associated with a significant increase in the incidence of ASD (fixed-effects model; $p$ $=0.33,0.39,0.83$; OR $=0.03,95 \% \mathrm{CI}[-0.03,0.10]$; OR = $1.47,95 \% \mathrm{CI}[0.61,3.51]$; $\mathrm{OR}=1.13,95 \% \mathrm{CI}[0.37,3.42]$, respectively, Fig. 8).

Pre- and post-operative L1-S1 sagittal vertical axis (SVA) Two studies (714of 2896 patients) [16, 22] reported preand post-operative L1-S1 SVA between ASD group and 


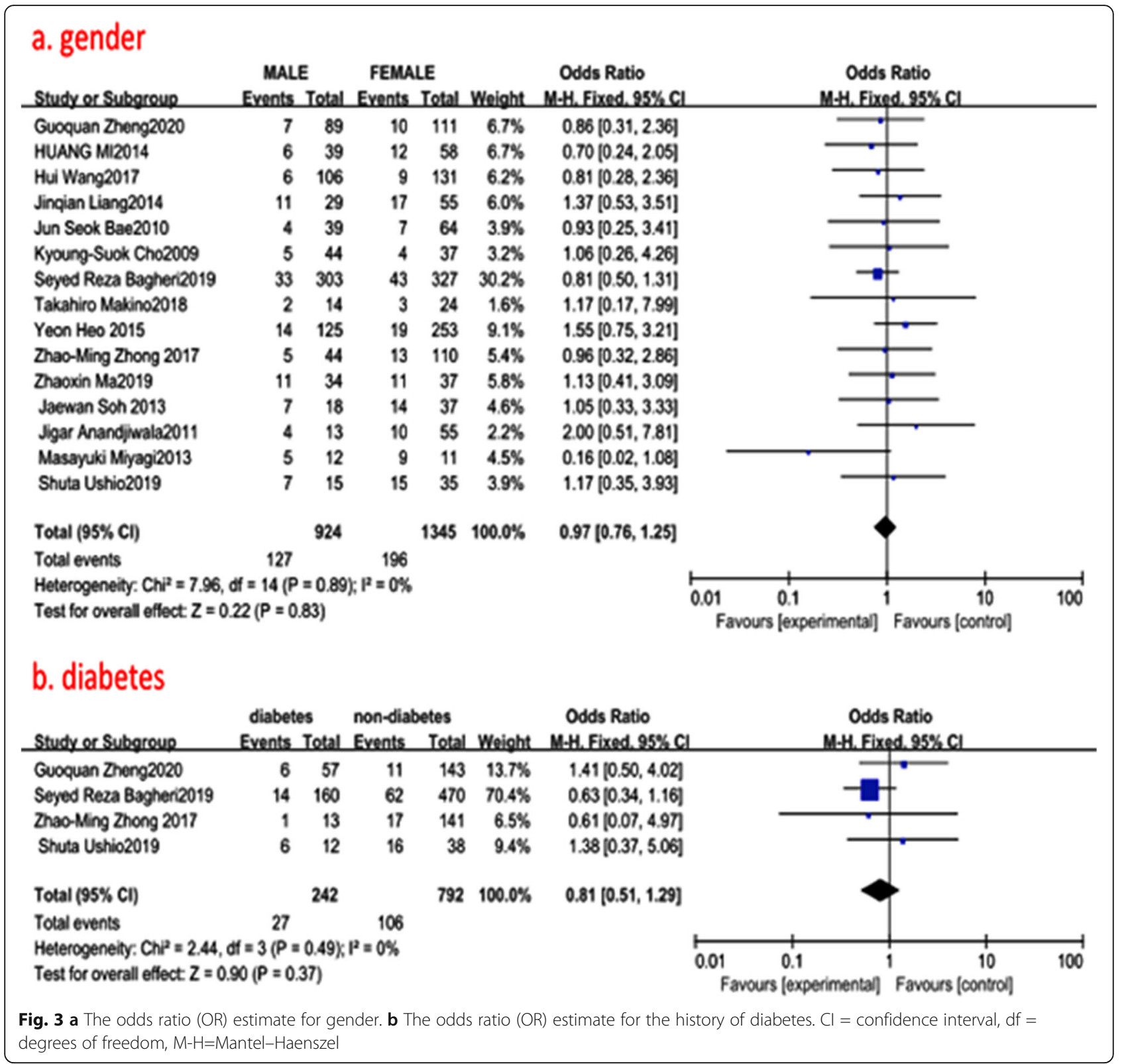

non-ASD group. There was no significance in the test for heterogeneity and the studies had low heterogeneity (two $p$ for heterogeneity $=0.65,0.84 ; I^{2}=0,0 \%$, Fig. 9). The meta-analysis showed that both pre- and postoperative L1-S1 SVA were associated with a significant increase in the incidence of ASD (fixed-effects model; $p<$ $0.00001, \mathrm{SMD}=6.94,95 \%$ CI $[4.85,9.03]$; $\mathrm{SMD}=3.87$, 95\% CI [2.33, 5.40], respectively, Fig. 9).

Pre- and post-operative pelvic tilt (PT)

Five studies (669 of 2896 patients) [19, 20, 22, 29, 30] reported pre- and post-operative PT between ASD group and non-ASD group. There was no significance in the test for heterogeneity and the studies had low heterogeneity (two $p$ for heterogeneity $=0.34,0.55 ; I^{2}$ $=11,0 \%$, Fig. 10). The meta-analysis showed that both pre- and post-operative PT were not associated with a significant increase in the incidence of ASD (fixed-effects model; $p=0.53,0.14, \mathrm{SMD}=-0.50$, $95 \%$ CI $[-2.06,1.06] ; \mathrm{SMD}=1.89,95 \%$ CI $[-0.59$, 4.36], respectively, Fig. 10).

Pre- and post-operative sacral slope (SS)

Eight studies (1492 of 2896 patients) [14, 16, 19, 20, $22,23,29,30]$ reported pre- and post-operative SS between ASD group and non-ASD group. There was 


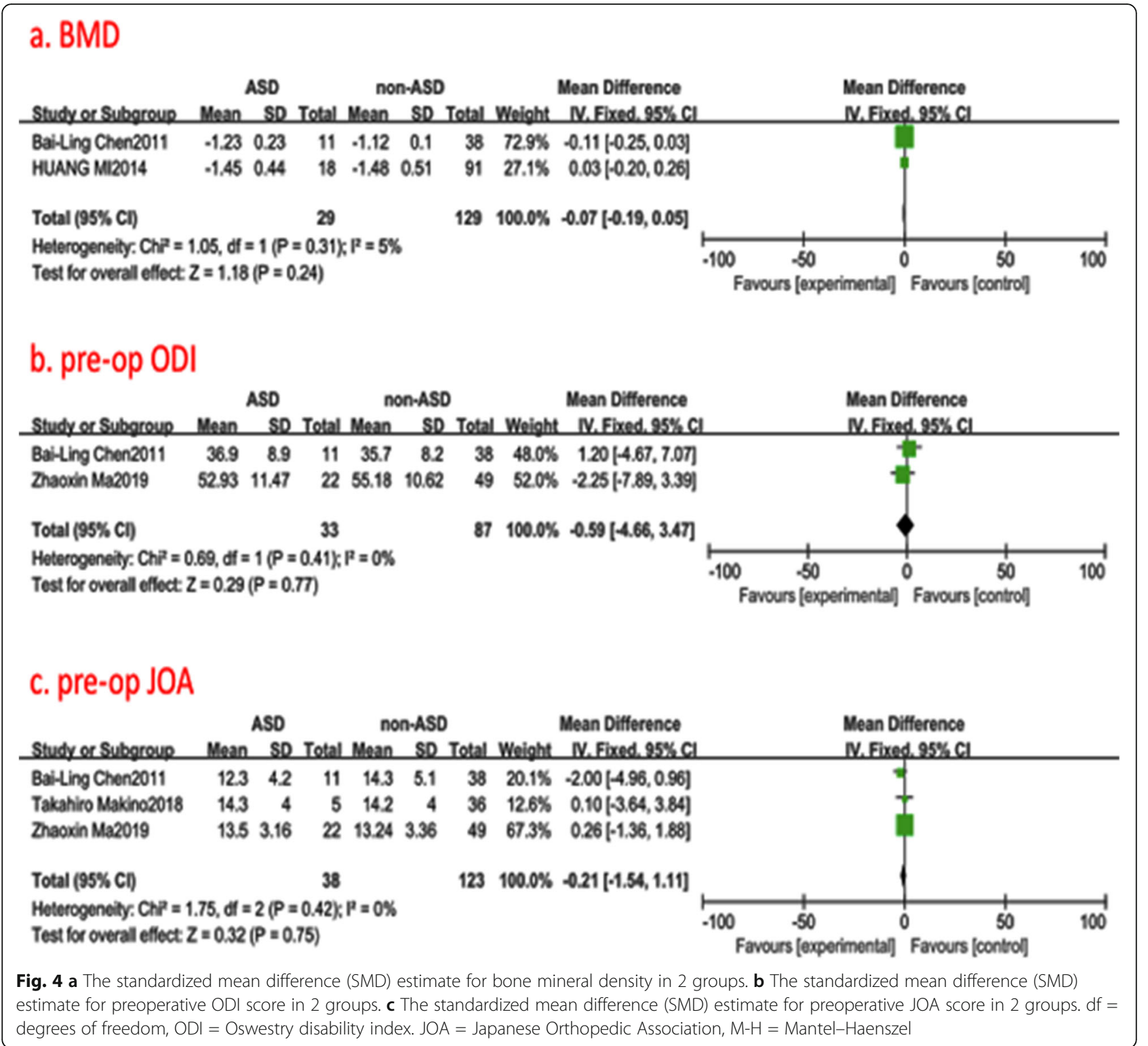

no significance in the test for heterogeneity and the studies had low heterogeneity (two $p$ for heterogeneity $=0.92,0.66 ; I^{2}=0,0 \%$, Fig. 11). The metaanalysis showed that both pre- and post-operative SS was not associated with a significant increase in the incidence of ASD (fixed-effects model; $p=0.07,0.21$, $\mathrm{SMD}=-1.27,95 \%$ CI $[-2.63,0.09] ; \mathrm{SMD}=-1.73$, 95\% CI [-4.42, 0.96], respectively, Fig. 11).

\section{Pre- and post-operative pelvic incidence (PI)}

Five studies (997 of 2896 patients) [18-20, 23, 30] reported pre- and post-operative PI between ASD group and non-ASD group. There was no significance in the test for heterogeneity and the studies had low heterogeneity (two $p$ for heterogeneity $=0.47,0.33 ; I^{2}=$ $0,0 \%$, Fig. 12). The meta-analysis showed that preoperative PI was associated with a significant increase in the incidence of ASD; however, post-operative PI was not associated with it (fixed-effects model; $p=0.02,0.67$, $\mathrm{SMD}=-2.13,95 \%$ CI $[-3.95,-0.30] ; \mathrm{SMD}=0.90,95 \%$ CI $[-3.19,4.99]$, respectively, Fig. 12).

Pre- and post-operative lumbar lordosis (LL)

Twelve studies (1423 of 2896 patients) [12, 14, 15, 17, 19-21, 23, 26, 28-30] reported pre- and post-operative LL between ASD group and non-ASD group. There was 


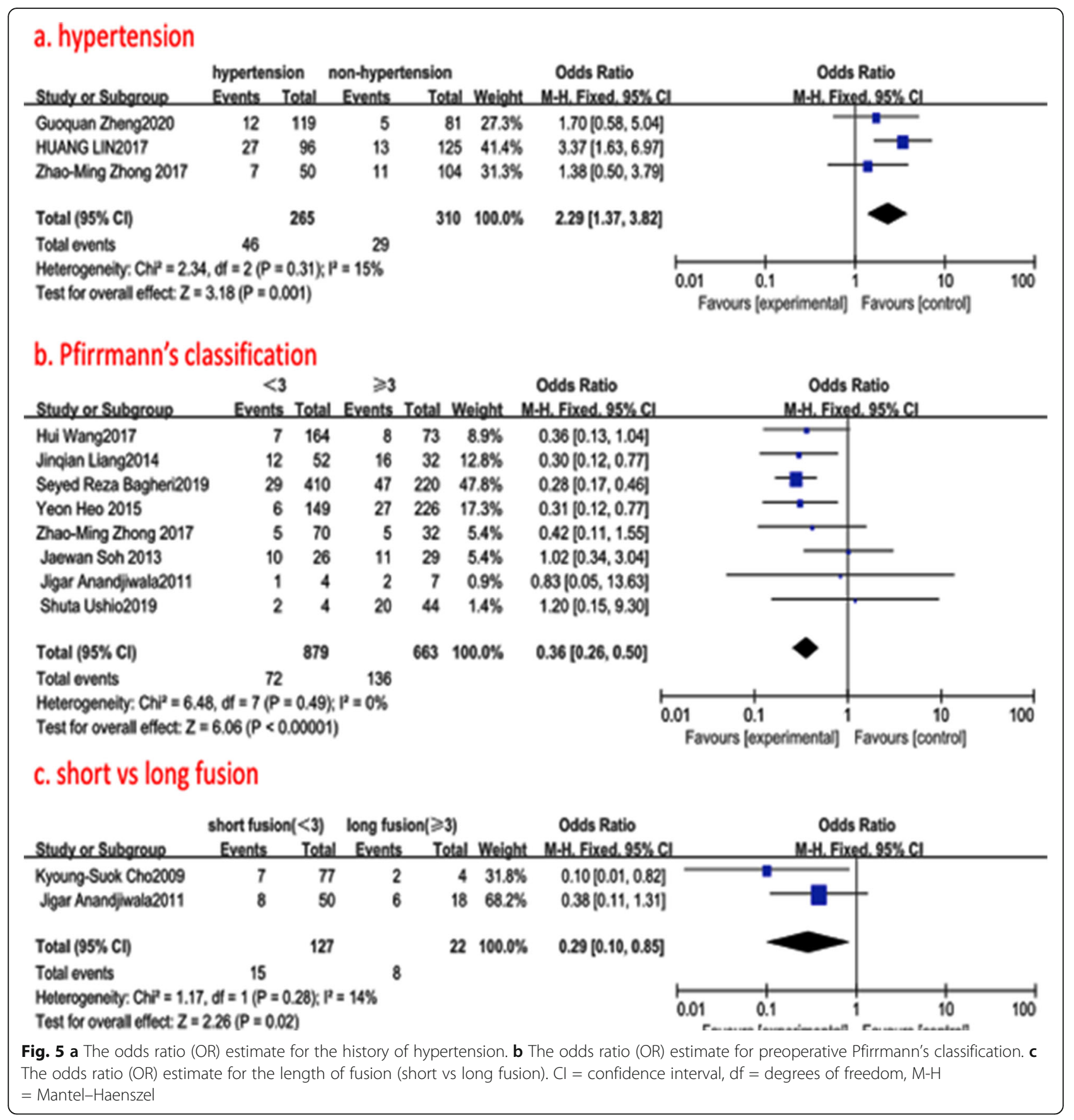

no significance in the test for heterogeneity and the studies had low heterogeneity (two $p$ for heterogeneity = $0.04,0.41 ; I^{2}=45,0 \%$, Fig. 13). The meta-analysis showed that preoperative LL was not associated with a significant increase in the incidence of ASD; however, post-operative LL was associated with it (fixed-effects model; $p=0.10,0.002$, SMD $=-0.75,95 \%$ CI $[-1.65$, $0.14] ;$ SMD $=-3.70,95 \% \mathrm{CI}[-5.99,-1.42]$, respectively, Fig. 13).
Publication bias

After detection of publication bias by STATA 12.0, there was no publication bias found for all included studies (all $p>0.05$ ).

\section{Discussion}

Degenerative lumbar diseases are common diseases in the clinic, especially in the elderly population. Posterior lumbar fusion surgery is a popular surgical 


\section{a. pre-op Superior facet violation}

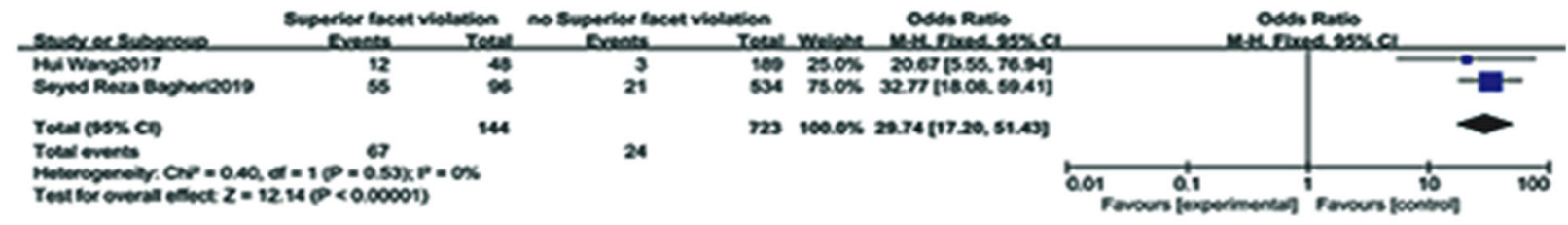

\section{b. pre-op Lumbosacral joint angle}

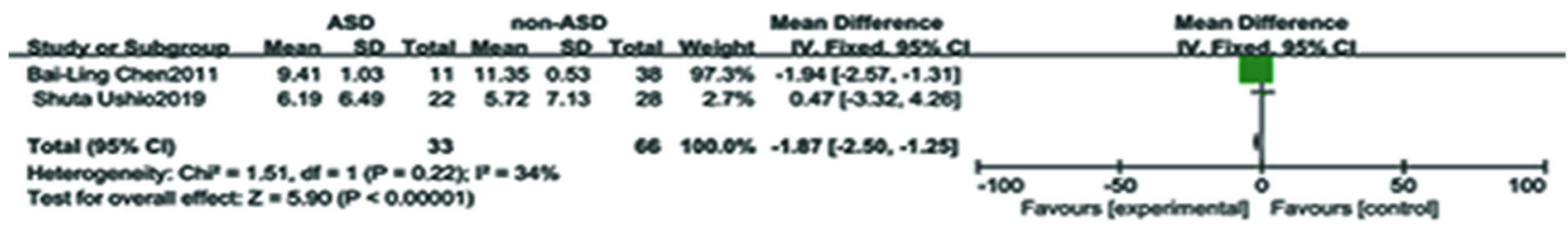

Fig. 6 a The odds ratio (OR) estimate for preoperative superior facet violation. b The standardized mean difference (SMD) estimate for preoperative lumbar-sacral joint angle in 2 groups. $\mathrm{Cl}=$ confidence interval, $\mathrm{df}=$ degrees of freedom, $\mathrm{M}-\mathrm{H}=$ Mantel-Haenszel

procedure in treatment for patients with degenerative spinal disorders. ASD, as a complication of posterior lumbar spinal fusion surgery, always attracts the attention of spine surgeons $[3,6,8,9]$. In 2004, Paul Park [31] reviewed articles involved ASD after lumbar fusion surgery and concluded that age, posterior lumbar interbody fusion, injury to the facet joint of the adjacent segment, long-segment fusion, sagittal alignment, pre-existing degenerated disc at the adjacent level, LL, osteoporosis, female gender, postmenopausal state were potential risk factors for ASD. Furthermore, in 2012, Brandon D [32] performed a mate-analysis on ASD indicating that age more than 60 years, male sex, facet degeneration, degenerative disc disease, adjacent to the fused segment, multilevel fusion, fusion to L5, and excessive disc height distraction. However, this article was limited to higherquality studies because of only 5 included studies. Although many scholars pay more attention to ASD after spine surgery, the risk factors associated with ASD are controversial.

Thus, we perform a meta-analysis to evaluate the risk factors associated with ASD [12-30]. The rate of ASD after posterior lumbar fusion surgery was $18.6 \%$ (ranged from 8.5 to $69.4 \%$ ) in this study. The pooled results from this meta-analysis suggested that gender of patients, history of diabetes, BMD, preoperative ODI and JOA, the type of fusion (PLIF vs TLIF), type of bone graft (auto- vs allograft), fusion to S1 (vs non-fusion to S1), diagnose (lumbar disc herniation, lumbar spinal stenosis, lumbar spondylolisthesis), preoperative PT, LL and SS, postoperative SS, PT, and PI were not were associated with a significant increase in the incidence of ASD. However, older age, BMI, the history of smoking and hypertension, preoperative adjacent disc degeneration, long-segment fusion, superior facet violation, high lumbosacral joint angle, pre- and post-operative L1-S1 SVA, post-operative LL, and preoperative PI were associated with a significant increase in the incidence of ASD.

Aota [33] observed that patients older than 55 years of age were at risk of ASD. However, some articles [12, 16, 19] indicated that age was not a significant factor of ASD. In our study, the older age demonstrated an increased risk of developing ASD. The reason that the older spine is less flexible and more difficult to adapt to the biomechanical alterations after fusion might partially explain this difference [34].

Bagheri [35] demonstrated that patients who had higher preoperative BMI showed a statistically increase in the risk of developing ASD. Wang [15] reported that BMI more than $25 \mathrm{~kg} / \mathrm{m}^{2}$ was found to be a risk factor for ASD. Our results were consistent with the previous studies $[12,15,35]$. In the present study, the history of smoking and hypertension was considered a risk of ASD, but the reason was unexplained.

Anandjiwala [26] demonstrated that preoperative disc degeneration at an adjacent level was a significant indicator of developing ASD. Our finding confirmed that patients with preoperative Pfirrmann's classification of more than 3 in the radiographic 


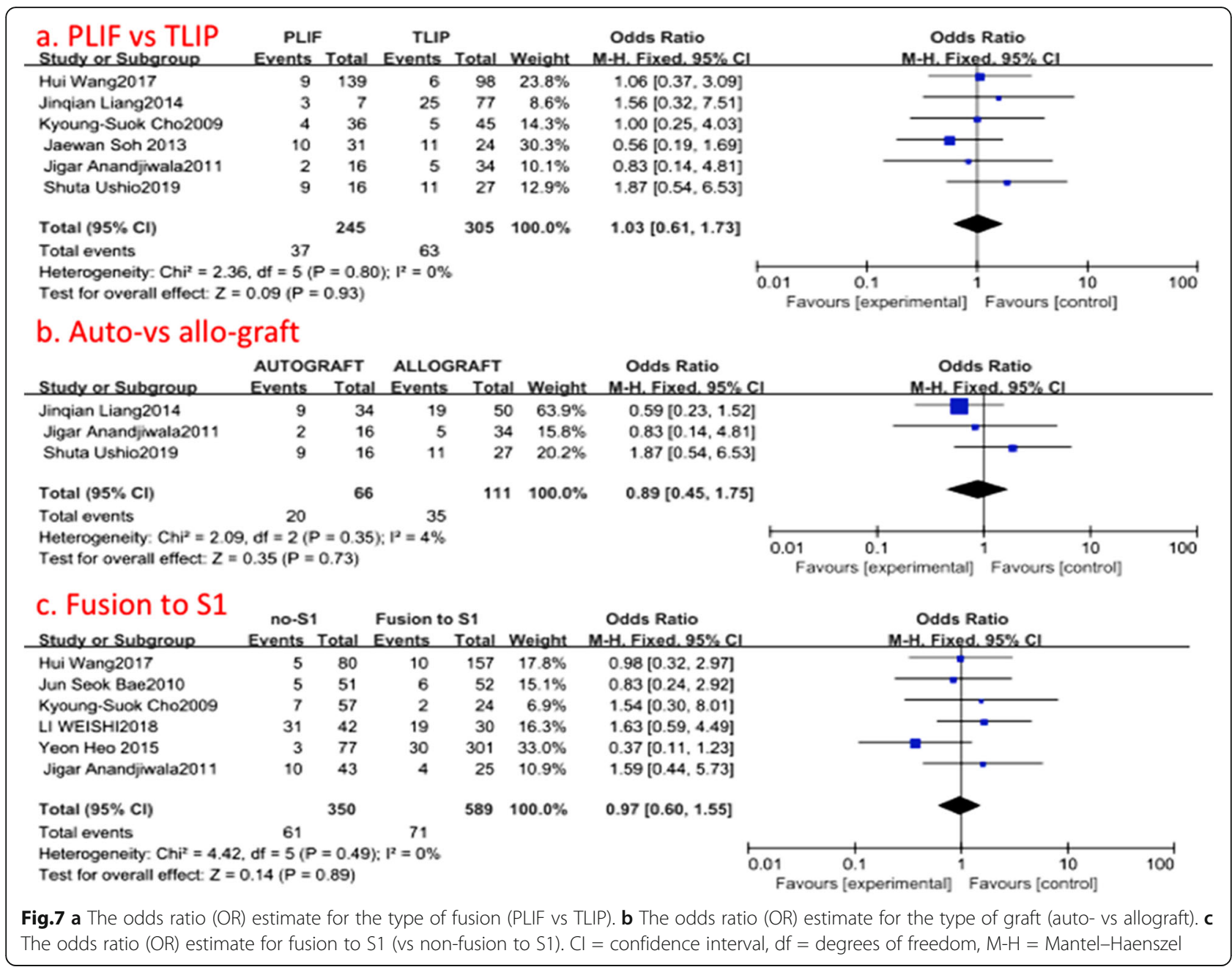

adjacent segment were found to be a statistic risk factor of ASD. Compared with patients whose Pfirrmann's classification was less than 3, biomechanical alterations caused by fusion make it more vulnerable to experience degeneration at the adjacent level that preoperative Pfirrmann's classification was more than 3. Additionally, we also found that preoperative superior facet violation was related to the increasing rate of ASD. Actually, preoperative superior facet violation is a form of degeneration at the adjacent segment, causing deduced adaptability to biomechanical change.

Long fusion that was more than 3 levels demonstrated a significant relationship with the incidence of ASD. Ghiselli et al. [36] reported that multiplelevel fusion had a three times higher risk for developing ASD than single-level fusion. Decreased elasticity and increased stiffness of the lumbar segment caused by long fusion are difficult to accommodate biomechanical changes at the adjacent motion segment including stress concentration and intradiscal pressures, which make it easier to experience degeneration at the adjacent segment.

It remains controversial as to whether an association exists between sagittal malalignment and ASD. Anandjiwala [26] showed that sagittal alignment parameters were not associated with the development of ASD. Zhong [19] demonstrated the same results. While other articles showed that patients with post-operative sagittal imbalance have a statistically significant increased chance of developing ASD [37, 38], our finding showed that partial sagittal parameters had a close relationship with the development of ASD. Djurasovic [39] found the patients developing ASD with a significantly lower level of LL. Wu [40] reported that the post-operative angle of LL was $7.9^{\circ}$ higher than the preoperative angle in patients after PLIF. Nakashima [41] concluded that appropriate post-operative LL after surgery could play a 


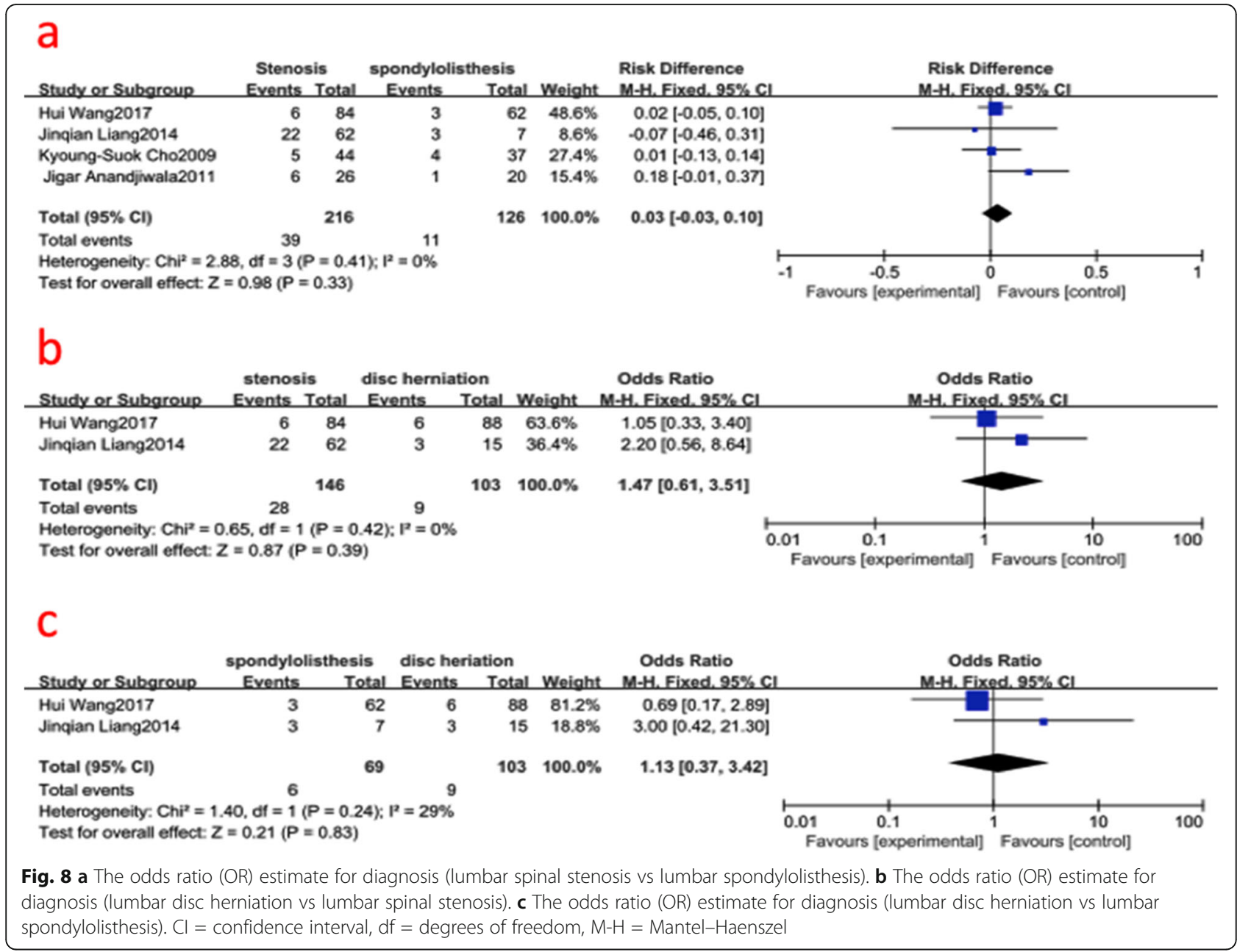

crucial role in the prevention of ASD. In the present study, post-operative LL was found to be a risk of ASD, while preoperative LL was not. Nakashima [42] believed that the patients with a high preoperative PI value have a significantly higher risk of ASD after spinal fusion, but he did not explore whether post-operative PI is the risk of ASD. In our study, regarding the role of preoperative PI to ASD, we were consistent with Nakashima. Nevertheless, post-operative PI was not associated with a significant increase in the incidence of ASD.

Kumar [43] showed that patients with a normal C7 plumb line alignment had a lower incidence of adjacentlevel change following lumbar spinal fusion. Liang [16] found that patients with normal post-operative lumbar sagittal alignment had a lower incidence of ASD. In addition, he concluded that the preoperative L1-S1 SVA was found to be a potential risk factor for predicting ASD after lumbar spine fusion. Our findings showed that both preoperative and post-operative L1-S1 SVA were associated with a significant increase in the incidence of ASD. Correction of sagittal alignment by spinal fusion plays an important role in the development of ASD. It ensures the proper conditions for fusion and facilitates the preservation of the adjacent segment. Abnormal sagittal alignment can have a deleterious effect on the adjacent segment.

There were several limitations to this study. First, we evaluated only radiological and asymptomatic ASD, but symptomatic one was not considered. Additionally, some factors had two included studies. The mentioned above might impact the accuracy of the results. Second, some factors, like PI-LL, might be risk factors for ASD. Because related studies were few and could not get pooled result, we excluded them. Third, follow-up time varied between the studies and thus may influence our results. Fourth, all the included studies come from Asian countries, which may affect the bias of results.

In conclusion, older age, BMI, the history of smoking and hypertension, preoperative adjacent disc degeneration, long-segment fusion, superior facet violation, high 


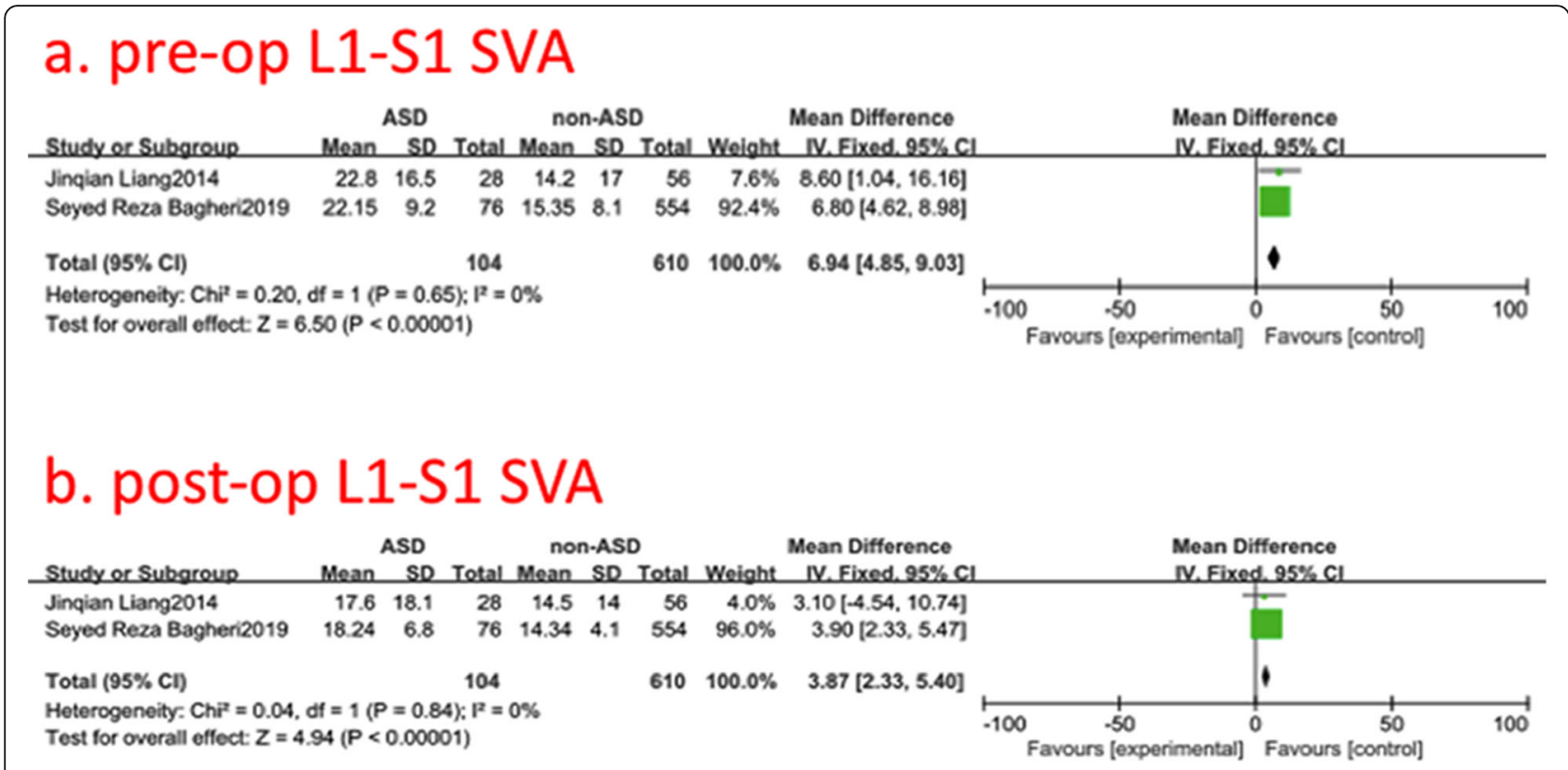

Fig. 9 a The standardized mean difference (SMD) estimate for preoperative L1-S1 sagittal vertical axis (SVA)in 2 groups. b The standardized mean difference (SMD) estimate for post-operative L1-S1SVA in 2 groups. Cl = confidence interval, $d f=$ degrees of freedom, M-H = Mantel-Haenszel

\section{a. pre-op PT}

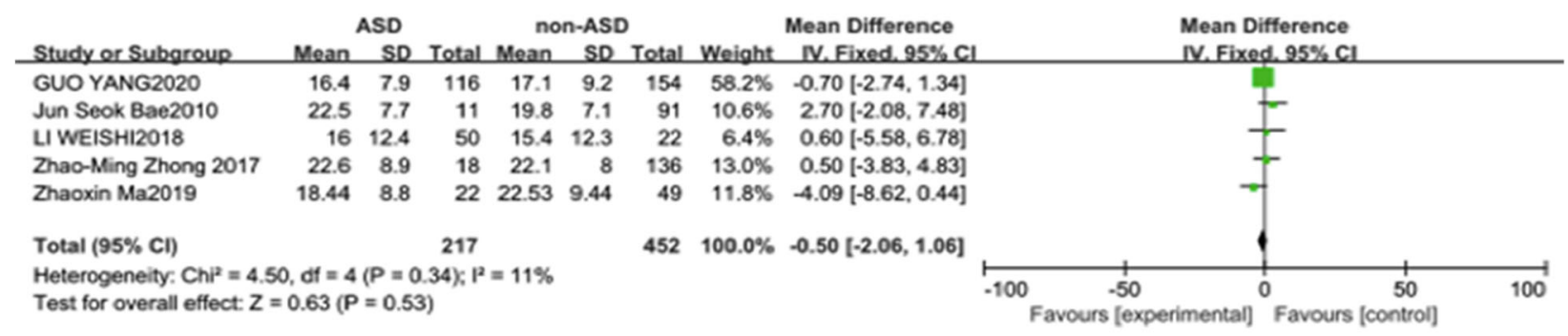

\section{b. post-op PT}

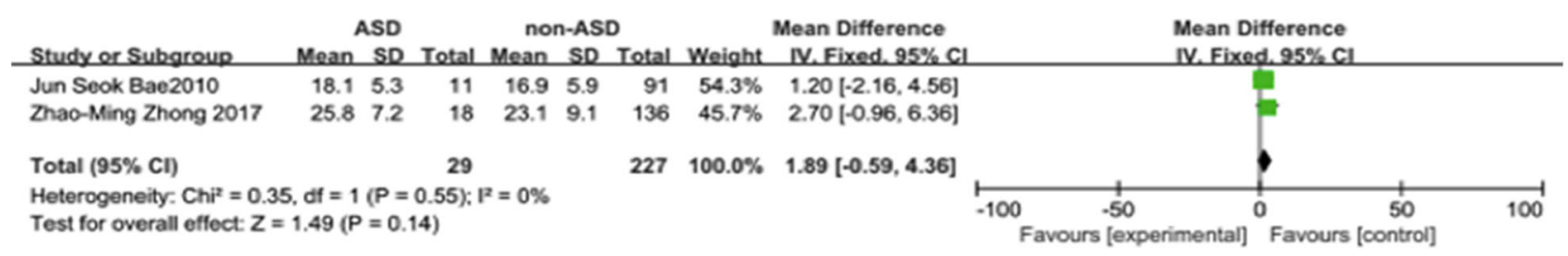

Fig. 10 a The standardized mean difference (SMD) estimate for preoperative pelvic tilt (PT)in 2 groups. $\mathbf{b}$ The standardized mean difference (SMD) estimate for post-operative PT in 2 groups. $\mathrm{Cl}=$ confidence interval, $\mathrm{df}=$ degrees of freedom, $\mathrm{M}-\mathrm{H}$ = Mantel-Haenszel 


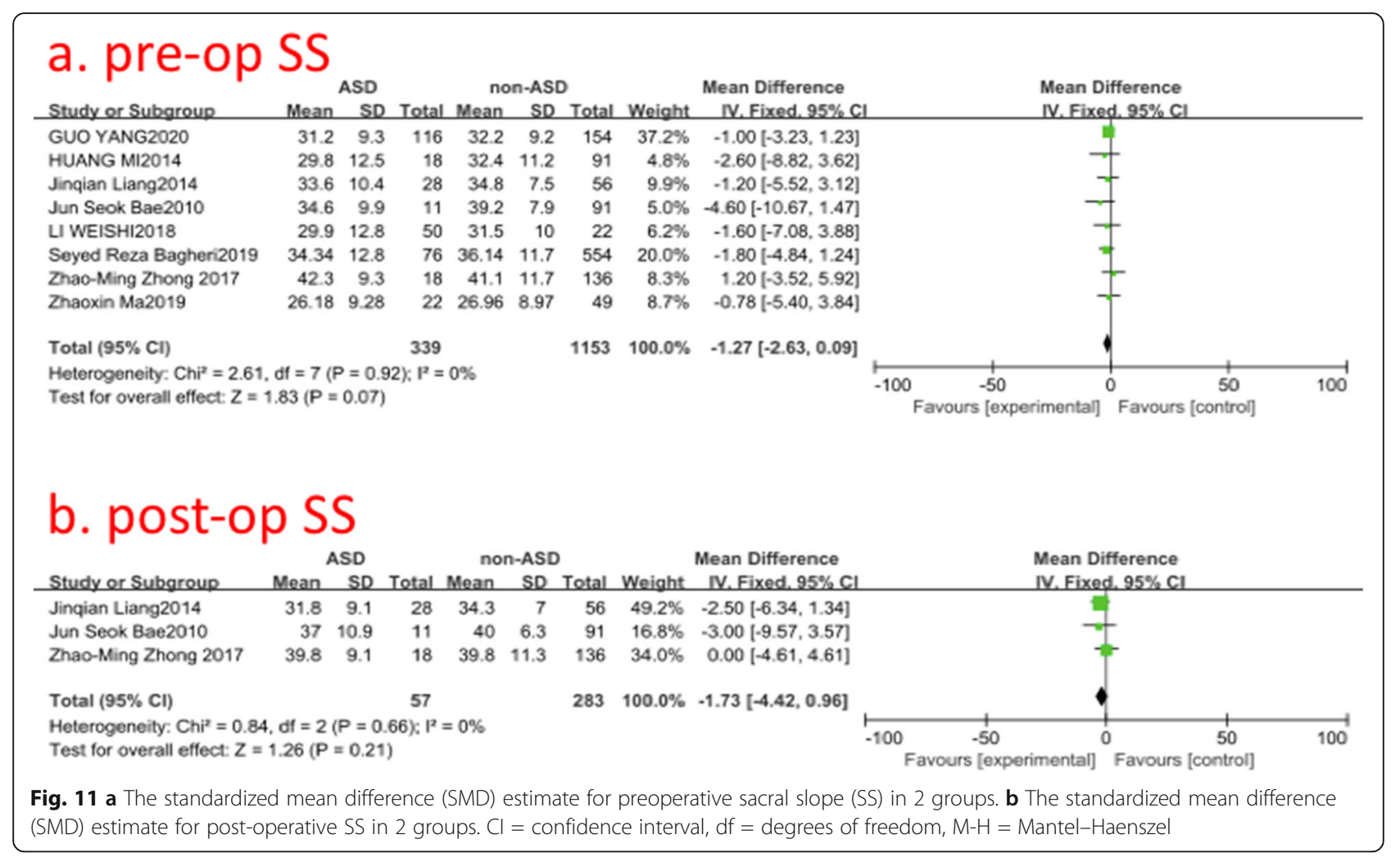

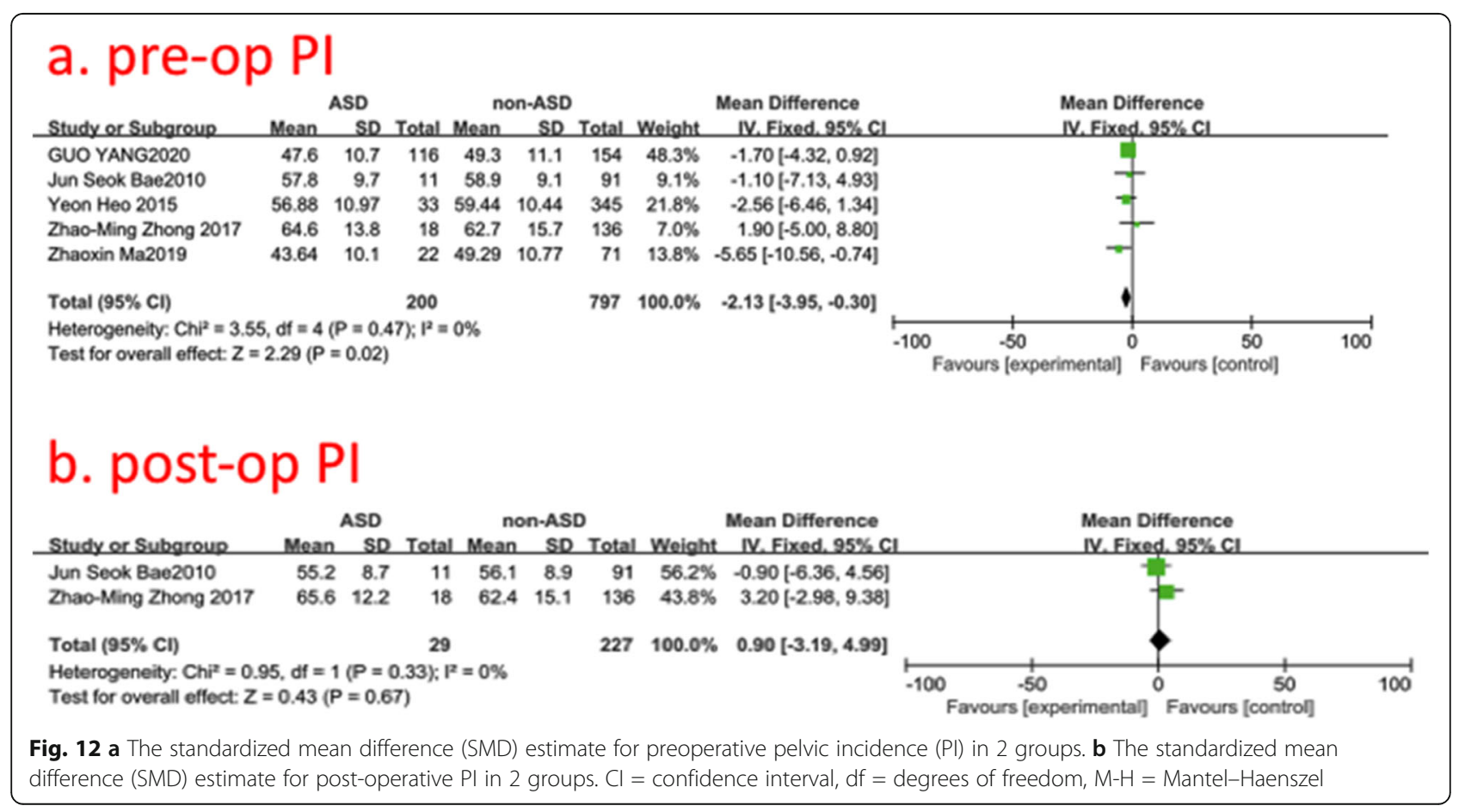




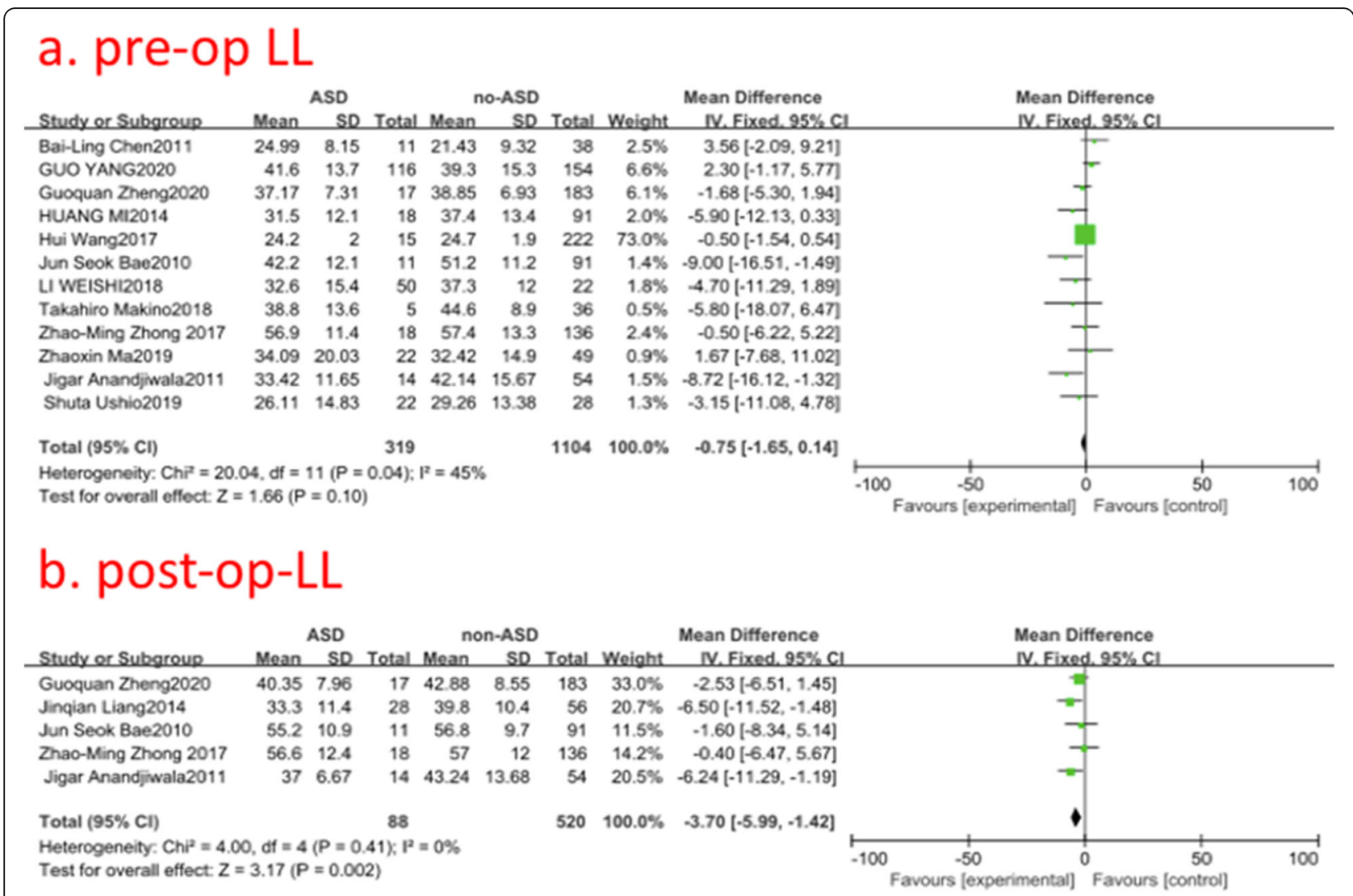

Fig. 13 a The standardized mean difference (SMD) estimate for preoperative lumbar lordosis (LL) in 2 groups. b The standardized mean difference (SMD) estimate for post-operative LL in 2 groups. $\mathrm{Cl}=$ confidence interval, $\mathrm{df}=$ degrees of freedom, $\mathrm{M}-\mathrm{H}=\mathrm{Mantel}-\mathrm{Haenszel}$

lumbosacral joint angle, pre- and post-operative L1-S1 SVA, post-operative LL, and preoperative PI were associated with a significant increase in the incidence of ASD. In this meta-analysis, we can clearly see which kind of people more likely had ASD after surgery. We hope this article can provide a reference for spinal surgeons in the treatment of lumbar degeneration diseases. Meanwhile, it is helpful for future study on ASD. Further large-scale, well-designed studies are urgently needed.

\section{Supplementary Information}

Supplementary information accompanies this paper at https://doi.org/10. 1186/s13018-020-02032-7.

\section{Additional file 1.}

\section{Abbreviations}

ASD: Adjacent segment degeneration; BMl: Body mass index; BMD: Bone mineral density; LL: Lumbar lordosis; PI: Pelvic incidence; PT: Pelvic tilt; SS: Sacral slope; SVA: Sagittal vertical axis

\section{Acknowledgements}

Not applicable.

\section{Authors' contributions}

Wang Tao collected data and write the essay; Ding Wenyuan is responsible for the design. The author (s) read and approved the final manuscript.
Availability of data and materials

Yes.

Ethics approval and consent to participate

Not applicable.

\section{Consent for publication}

Yes.

Competing interests

There are no competing interests.

Author details

${ }^{1}$ Wuxi No.9 People Hospital, Wuxi, China. ${ }^{2}$ Department of Spinal Surgery, The Third Hospital of Hebei Medical University, No. 139 Ziqiang Road,

Shijiazhuang 050051, China.

Received: 27 May 2020 Accepted: 20 October 2020

Published online: 03 December 2020

\section{References}

1. Cheh G, Bridwell KH, Lenke LG, et al. Adjacent segment disease following lumbar/thoracolumbar fusion with pedicle screw instrumentation: a minimum 5-year follow-up. Spine. 2007:32(20):2253-7.

2. Park P, Garton HJ, Gala VC et al. Adjacent segment disease after lumbar or lumbosacral fusion: review of the literature. Spine (Phila Pa 1976). 2004 29: 1938-1944. doi: 00007632-200409010-00019[pii].

3. Lee CS, Hwang CJ, Lee SW, et al. Risk factors for adjacent segment disease after lumbar fusion. Eur Spine J. 2009;11:1637-43.

4. Quinell RC, Stockdale HR. Some experimental observations on the influence of a single floating fusion on the remaining lumbar spine. Spine. 1981;6:263-7. 
5. Battie MG, Videman T, Parent E. Lumbar disc degeneration: epidemiology and genetics influences. Spine. 2004:29:2679-90.

6. Puvanesarajah V, Cancienne JM, Werner BC, et al. Perioperative complications associated with posterolateral spine fusions: a study of elderly medicare beneficiaries. Spine J. 2016;16:S266-7 [Epub ahead of print].

7. Park P, Garton HJ, Gala VC, et al. Adjacent segment disease after lumbar or lumbosacral fusion: review of the literature. Spine (Phila Pa 1976). 2004;29: 1938-44.

8. Kumar MN, Baklanov A, Chopin D. Correlation between sagittal plane changes and adjacent segment degeneration following lumbar spine fusion. Eur Spine J. 2001;10:314-9.

9. Etebar S, Cahill DW. Risk factors for adjacent-segment failure following lumbar fixation with rigid instrumentation for degenerative instability. J Neurosurg. 1999;90:163-9.

10. Ha KY, Chang CH, Kim KW, Kim YS, Na KH, Lee JS. Expression of estrogen receptor of the facet joints in degenerative spondylolisthesis. Spine (Phila Pa 1976). 2005;30:562-6 00007632-200503010-00015[pii].

11. Xiao SW, Jiang $H$, Yang LJ, Xiao ZM. Anterior cervical discectomy versus corpectomy for multilevel cervical spondylotic myelopathy: a meta-analysis. Eur Spine J. 2015;24:31-9. https://doi.org/10.1007/s00586-014-3607-1.

12. Zheng G, Wang C, Wang $T$, et al. Relationship between postoperative lordosis distribution index and adjacent segment disease following L4-S1 posterior lumbar interbody fusion. J Orthop Surg Res. 2020;15(1):129. https://doi.org/10.1186/s13018-020-01630-9.

13. Lin $H$, Zhaopeng $C$, Keng $C$, et al. Retrospective analysis of preoperative risk factors of adjacent segment degeneration after posterior lumbar interbody fusion. J Clin Orthop Res. 2017;2(1):1-5.

14. $\mathrm{Mi} \mathrm{H}, \mathrm{Yu} \mathrm{M}$, Xiaoguang $\mathrm{L}$, et al. Correlative factors for adjacent segment degeneration after lumbar spinal fusion. Chin J Spine Spinal Cord. 2014; 24(3):199-203.

15. Wang $H, M a L$, Yang $D$, et al. Incidence and risk factors of adjacent segment disease following posterior decompression and instrumented fusion for degenerative lumbar disorders. Medicine (Baltimore). 2017;96(5):e6032. https://doi.org/10.1097/MD.0000000000006032.

16. Liang J, Dong Y, Zhao H. Risk factors for predicting symptomatic adjacent segment degeneration requiring surgery in patients after posterior lumbar fusion. J Orthop Surg Res. 2014;9:97. https://doi.org/10.1186/s13018-014-0097-0.

17. Makino T, Honda H, Fujiwara H, Yoshikawa H, Yonenobu K, Kaito T. Low incidence of adjacent segment disease after posterior lumbar interbody fusion with minimum disc distraction: A preliminary report. Medicine (Baltimore). 2018;97(2):e9631. https://doi.org/10.1097/MD.0000000000009631.

18. Heo Y, Park JH, Seong HY, et al. Symptomatic adjacent segment degeneration at the L3-4 level after fusion surgery at the L4-5 level: evaluation of the risk factors and 10-year incidence. Eur Spine J. 2015:24(11) https://doi.org/10.1007/s00586-015-4188-3

19. Zhong ZM, Deviren V, Tay B, et al. Adjacent segment disease after instrumented fusion for adult lumbar spondylolisthesis: incidence and risk factors. Clin Neurol Neurosurg. 2017;2017(156):29-34. https://doi.org/10. 1016/j.clineuro.2017.02.020

20. Ma Z, Huang S, Sun J, Li F, Sun J, Pi G. Risk factors for upper adjacent segment degeneration after multi-level posterior lumbar spinal fusion surgery. J Orthop Surg Res. 2019;14(1):89. https://doi.org/10.1186/s13018-019-1126-9.

21. Ushio S, Hirai T, Yoshii T, et al. Preoperative risk factors for adjacent segment degeneration after two-level floating posterior fusion at L3-L5. Spine Surg Relat Res. 2019;4(1):43-9. https://doi.org/10.22603/ssrr.2019-0003.

22. Bagheri SR, Alimohammadi E, Froushani AZ, et al. Adjacent segment disease after posterior lumbar instrumentation surgery for degenerative disease: Incidence and risk factors. J Orthop Surg. 2019;27(2):1-6.

23. Bae JS, Lee S-H, Kim J-S, et al. Adjacent segment degeneration after lumbar interbody fusion with percutaneous pedicle screw fixation for adult lowgrade isthmic spondylolisthesis: minimum 3 years of follow-up. Neurosurgery. 2010;67:1600-8. https://doi.org/10.1227/NEU. Ob013e3181f91697.

24. Cho K-S, Kang S-G, Yoo D-S, et al. Risk factors and surgical treatment for symptomatic adjacent segment degeneration after lumbar spine fusion. J Korean Neurosurg Soc. 2009:46:425-30.

25. Soh J, Lee JC, Shin BJ. Analysis of risk factors for adjacent segment degeneration occurring more than 5 years after fusion with pedicle screw fixation for degenerative lumbar spine. Asian Spine J. 2013;7(4):273-81.

26. Anandjiwala J, Seo J-Y, Ha K-Y, et al. Adjacent segment degeneration after instrumented posterolateral lumbar fusion: a prospective cohort study with a minimum five-year follow-up. Eur Spine J. 2011;20:1951-60. https://doi. org/10.1007/s00586-011-1917-0

27. Miyagi M, Ikeda $\mathrm{O}$, Ohtori $\mathrm{S}$, et al. Additional decompression at adjacent segments leads to adjacent segment degeneration after PLIF. Eur Spine J. 2013;22:1877-83. https://doi.org/10.1007/s00586-013-2694-8.

28. Chen B-L, Wei F-X, Ueyama K, et al. Adjacent segment degeneration after single-segment PLIF: the risk factor for degeneration and its impact on clinical outcomes. Eur Spine J. 2011;20:1946-50. https://doi.org/10.1007/ s00586-011-1888-1.

29. Weishi L, Zhouran S, Guo Y, et al. Effect of spinopelvic sagittal alignment on the development of adjacent segment degeneration after posterior lumbar fusion: investigation on cases with a minimum of 6 years of follow up. Chin J Spine Spinal Cord. 2018;28(10):865-72.

30. Guo Y, Zhouran S, Siyu Z, et al. The effect of pre-existing degeneration at adjacent segment on postoperative adjacent segment degeneration and surgical clinical outcome. Spine Spinal Cord. 2020;30(2):103-10.

31. Park P, Hugh J. Garton, et al. Adjacent segment disease after lumbar or lumbosacral fusion: review of the literature. SPINE. 2004;29(17):1938-44.

32. Lawrence BD, Wang J, et al. Predicting the risk of adjacent segment pathology after lumbar fusion. SPINE. 2012;37(22S):S123-32..

33. Aota Y, Kumano K, Hirabayashi S. Postfusion instability at the adjacent segments after rigid pedicle screw fixation for degenerative lumbar spinal disorders. J Spinal Disord. 1995;8:464-73.

34. Kim YJ, Bridwell KH, Lenke LG, Glattes CR, Rhim S, Cheh G. Proximal junctional kyphosis in adult spinal deformity after segmental posterior spinal instrumentation and fusion: minimum five-year follow-up. Spine. 2008;33(20):2179-84. https://doi.org/10.1097/BRS.0b013e31817c0428.

35. Bagheri SR, Alimohammadi E, Zamani Froushani A, et al. Adjacent segment disease after posterior lumbar instrumentation surgery for degenerative disease: incidence and risk factors. J Orthop Surg (Hong Kong). 2019;27: 2309499019842378.

36. Ghiselli G, Wang JC, Bhatia NN, Hsu WK, Dawson EG. Adjacent segment degeneration in the lumbar spine. J Bone Joint Surg Am. 2004;86-A:1497-503.

37. Zencica P, Chaloupka R, Hladikova J, et al. Adjacent segment degeneration after lumbosacral fusion in spondylolisthesis: a retrospective radiological and clinical analysis. Acta Chir Orthop Traumatol Cech. 2010;77:124-30.

38. Chung KJ, Suh SW, Swapnil K, et al. Facet joint violation during pedicle screw insertion: a cadaveric study of the adult lumbosacral spine comparing the two pedicle screw insertion techniques. Int Orthop. 2007;31:653-6.

39. Nakashima H, Kawakami N, Tsuji T, et al. Adjacent segment disease afterposterior lumbar interbody fusion: based on cases with a minimum of 10 years of follow-up. Spine (Phila Pa 1976). 2015;40:E831-41.

40. Wu CH, Wong $\mathrm{CB}$, Chen $\mathrm{LH}$, et al. Instrumented posterior lumbar interbodyfusion for patients with degenerative lumbar scoliosis. J Spinal Disord Tech. 2008;21:310-5.

41. Djurasovic MO, Carreon LY, Glassman SD, et al. Sagittal alignment as a riskfactor for adjacent level degeneration: a case-control study. Orthopedics. 2008:31:546.

42. Nakashima H, Kawakami N, Tsuji T, et al. Adjacent segment disease afterposterior lumbar interbody fusion: based on cases with a minimum of 10 years of follow-up. Spine. 2015;40(14):E831-41.

43. Kumar MN, Baklanov A, Chopin D. Correlation between sagittal planechanges and adjacent segment degeneration following lumbar spinefusion. Eur Spine J. 2001;10(4):314-9.

\section{Publisher's Note}

Springer Nature remains neutral with regard to jurisdictional claims in published maps and institutional affiliations. 